\title{
Hybrid IEEE 802.15.6 Wireless Body Area Networks Interference Mitigation Model for High Mobility Interference Scenarios
}

\author{
Anthony Mile, George Okeyo, Ann Kibe \\ School of Computing and Information Technology, Jomo Kenyatta University of Agriculture and Technology, Nairobi, Kenya \\ Email: mileanthony@yahoo.com, gokeyo@jkuat.ac.ke, annkibe@icsit.jkuat.ac.ke
}

How to cite this paper: Mile, A., Okeyo, G. and Kibe, A. (2018) Hybrid IEEE 802.15.6 Wireless Body Area Networks Interference Mitigation Model for High Mobility Interference Scenarios. Wireless Engineering and Technology, 9, 34-48. https://doi.org/10.4236/wet.2018.92004

Received: March 21, 2018

Accepted: April 27, 2018

Published: April 30, 2018

Copyright (c) 2018 by authors and Scientific Research Publishing Inc. This work is licensed under the Creative Commons Attribution International License (CC BY 4.0).

http://creativecommons.org/licenses/by/4.0/

\begin{abstract}
The field of Wireless Sensor Networks (WSNs) has revolutionized tremendously in the recent past with its major application in Wireless Body Area Networks (WBANs). This has in the same dimension attracted immense interests from the researchers and technology providers. The operational modality of the WBANs is that a few sensor nodes are placed in or around the body and that they are meant to operate within a limited condition while providing high performance in terms of WBAN life time, high throughput, high data reliability, minimum or no delay and low power consumption. As most of the WBAN operates within the universal Industrial, Scientific and Medical (ISM) Narrow Band (NB) wireless band (2.4 $\mathrm{Ghz}$ ) frequency band, this has posed a challenge in respect to inter, intra and co-channel interference especially in dense areas and high mobility scenarios. As well the body posture changes dynamically due to these mobility effects. In this paper, we propose a hybrid WBAN interference mitigation model based on Carrier Sense Multiple Access with Collision Avoidance (CSMA/CA) Contention Window (CW) approach and User Priority (UP) queues. Using Omnet++ simulation, a comparison to the IEEE 802.15.6 based WBAN protocol is presented under the standing, walking sitting and Lying postural mobility scenarios. The results show that the proposed hybrid model outperforms IEEE 802.15 .6 based CSMA/CA protocol in areas of network throughput, bandwidth efficiency and network delay in these mobility postures.
\end{abstract}

\section{Keywords}

IEEE 802.15.6, Interference Mitigation, WBAN Mobility, Priority Queues, CSMA/CA 


\section{Introduction}

Wireless sensor networks (WSN) is a technology which consists of spatially distributed autonomous sensors to monitor physical or environmental conditions, such as temperature, sound, pressure, etc. and to cooperatively pass their data through the network to a main location [1]. It incorporates a gateway that provides a wireless connectivity back to the wired world.

A rapidly growing application area of WSN is in the body area networks (BANs) which is used widely in the healthcare applications. The BANs have since developed from the IEEE 802.15.4 general WSN technology standard [2]. But due to the special needs of the healthcare applications, a special BAN technology called the wireless body area sensor networks (WBANs) was developed to majorly operate as the IEEE 802.15.6 standard as the IEEE 802.15.4 has been retained to operate in legacy WSN such as in industrial applications and other large scale environmental monitoring systems which can scale up to $100 \mathrm{~m}$ in radius. The WBANs was intended only to address special needs of the wireless body area sensor networks such as low power, low cost, low complexity, high throughput and short range wireless communication in and around the human body. Table 1 shows the differences between Wireless Sensor Networks and Wireless Body Area Networks.

Unlike the WSN where the sensor nodes are distributed in a wide area, WBAN consist of a small number of sensor nodes (most often about six) that are placed in or around the human body for remote monitoring. Figure 1 shows some of the different wireless sensor nodes in or around the body. Several sensors are placed in clothes, directly on the body or under the skin of a person and measure the temperature, blood pressure, heart rate, ECG, EEG, respiration rate etc. Since the sensor monitoring includes lifesaving human body signs which may determine between life and death for the patient, high reliability is expected in both the sensing and data transmission [3]. Unfortunately, performance of these WBANs decreases in high interference scenario's such as densely populated areas and in the ISM wireless band as this frequency band has co-channel interference from other technologies using the same frequency, such as IEEE 802.15.1 (Bluetooth), IEEE 802.11 (wireless fidelity - Wi-Fi) and IEEE 802.15.4 (zigbee). As opposed to the previous WSN used for WBAN such as IEEE 802.15.4, the IEEE 802.15.6 addresses these special needs for WBAN and is required to function properly within the transmission range of up to 5 meters when up to 10 WBANs are co-located [4]. Thus, there is as well high possibility of interference amongst WBANs operating in close range and other technologies as well. This is because of the mobility of the human body and the activity of the different body parts such as legs, head, arms among others which causes intra and inter-WBAN interference. Figure 2 shows an interference scenario in wireless body area networks.

Our proposed model is a hybrid WBAN Interference mitigation model which makes use of the CSMA/CA CW technique and User Priority (UP) queues technique 
to enable allocation of high priority to emergency critical sensor data and the rest of data is given lower priorities accordingly. We have used Omnet++ with Mixim framework, which makes simulation, more realistic and reliable [6]. MoBAN mobility model [7] [8] is used to establish the walking, standing, sitting and lying down postures for human body.

Table 1. Schematic overview of differences between wireless sensor networks and wireless body area networks [5].

\begin{tabular}{|c|c|c|}
\hline Challenges & Wireless Sensor Network & Wireless Body Area Sensor Network \\
\hline Scale & $\begin{array}{l}\text { Monitored environment } \\
\text { (meters/kilometers) }\end{array}$ & Human body (centimeters/meters) \\
\hline Node Number & $\begin{array}{l}\text { Many redundant nodes for wide area } \\
\text { coverage }\end{array}$ & Fewer, limited in space \\
\hline Result accuracy & Through node redundancy & $\begin{array}{l}\text { Through node accuracy and } \\
\text { robustness }\end{array}$ \\
\hline Node Tasks & Node performs a dedicated task & Node performs multiple tasks \\
\hline Node Size & Small is preferred, but not important & Small is essential \\
\hline Network Topology & Very likely to be fixed or static & More variable due to body movement \\
\hline Data Rates & Most often homogeneous & Most often heterogeneous \\
\hline Node Replacement & $\begin{array}{l}\text { Performed easily, nodes even } \\
\text { disposable }\end{array}$ & $\begin{array}{l}\text { Replacement of implanted nodes } \\
\text { difficult }\end{array}$ \\
\hline Node Lifetime & Several years/months & $\begin{array}{l}\text { Several years/months, smaller battery } \\
\text { capacity }\end{array}$ \\
\hline Power Supply & $\begin{array}{l}\text { Accessible and likely to be replaced } \\
\text { more easily and frequently }\end{array}$ & $\begin{array}{l}\text { Inaccessible and difficult to replace in } \\
\text { an implantable setting }\end{array}$ \\
\hline Power Demand & Likely to be large, energy supply easier & $\begin{array}{l}\text { Likely to be lower, energy supply } \\
\text { more difficult }\end{array}$ \\
\hline $\begin{array}{l}\text { Energy Scavenging } \\
\text { Source }\end{array}$ & Most likely solar and wind power & $\begin{array}{l}\text { Most likely motion (vibration) and } \\
\text { thermal (body heat) }\end{array}$ \\
\hline Biocompatibility & $\begin{array}{l}\text { Not a consideration in most } \\
\text { applications }\end{array}$ & $\begin{array}{l}\text { A must for implants and some } \\
\text { external sensors }\end{array}$ \\
\hline Security Level & Lower & $\begin{array}{l}\text { Higher, to protect patient } \\
\text { information }\end{array}$ \\
\hline Impact of Data Loss & $\begin{array}{l}\text { Likely to be compensated by } \\
\text { redundant nodes }\end{array}$ & $\begin{array}{l}\text { More significant, may require } \\
\text { additional measures to ensure QoS } \\
\text { and real-time data delivery. }\end{array}$ \\
\hline Wireless Technology & Bluetooth, ZigBee, GPRS, WLAN, ... & Low power technology required \\
\hline Node Tasks & Node performs a dedicated task & Node performs multiple tasks \\
\hline Node Size & Small is preferred, but not important & Small is essential \\
\hline Network Topology & Very likely to be fixed or static & More variable due to body movement \\
\hline
\end{tabular}




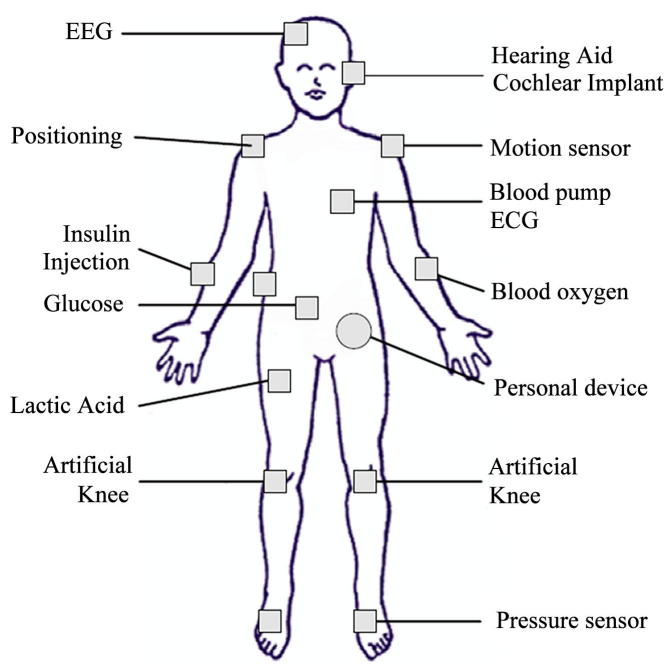

Figure 1. Example of patient monitoring in a WBAN.

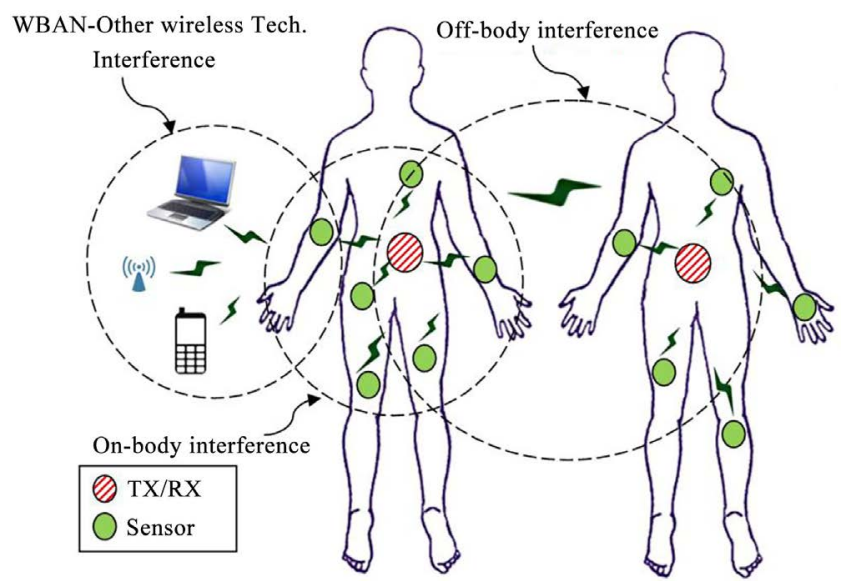

Figure 2. Interference in WBANs.

The rest of the paper is organized as follows: In Section 2, we present an Overview of IEEE 802.15.6 WBAN standard, Section 3 we discuss the related work, Section 4 we present the motivation behind this work while the proposed model is described in section 5, the performance parameters are presented in Section 6, Section 7 we present the performance analysis results and conclude our findings in Section 8.

\section{The IEEE 802.15.6 WBAN Standard}

The IEEE standards related to the technical requirements of WBANs includes support for quality of service (QoS), extremely low power, highly reliability wireless communication (low latencies and data loss) and data rates up to 10 Mbps [9] [10]. The WBAN consists of a few sensors communicating with a gateway device to the internet. A typical medical WBAN is stated to have 6 nodes and scalable to 256 nodes. IEEE 802.15.6 Draft, 2010 stated the requirements of WBANs as operating range of $3 \mathrm{~m}$ for WBANs of upto 10 piconets per person and supporting 256 nodes in each net within $6 \mathrm{~m}^{3}$ [11] [12] [13]. As well Mo- 
vassaghi et al. [14] states that the requirements of WBANs based on the IEEE 802.15.6 standard are: the bit rate of a link is in the range of $10 \mathrm{kbps}$ to $10 \mathrm{Mbps}$, packet error rate should be less than $10 \%$ for a 256 octet payload for $95 \%$ of links, and the time to join or leave a network should be less than 3s. Also according to the IEEE 802.16 standard, the nodes can be organised into one or two-hop star WBANs with a single coordinator or hub controlling the entire operation of each of the WBAN [15].

The WBAN operates different frequency bands in different countries [16]. A summary of some of the frequency bands available for WBAN in different countries are shown in Figure 3. Medical Implant Communications Service (MICS) band is a licensed band used for implant communication and has the same frequency range (402 - $405 \mathrm{MHz}$ ) in most of the countries. Wireless Medical Telemetry Services (WMTS) is a licensed band used for medical telemetry system. Both of the MICS and WMTS bandwidths do not support high data rate applications. The Industrial, Scientific and Medical (ISM) band in $2.4 \mathrm{Ghz}$ frequency range supports high data rate applications and is also available worldwide. However, the chances of interference are very high as many wireless devices including IEEE 802.1 and IEEE802.15.4 operate at the same ISM band.

\section{The WBAN Mobility}

The sensor nodes embedded or implanted on or in a human body exhibit mobility as a result of human body movement and the movement of the different body parts like the arm, leg, among others causing nodal mobility.

The mobility model used is very important and plays a significant role in evaluating the performance of the WBAN. In this study, we adopt the MoBAN mobility model as the most ideal model for WBAN. The ability of the MoBAN mobility model to support both the postural selector and group mobility makes it good for WBAN.

The posture selector determines the current posture at any given time. The posture selections are defined as lying down, walking, standing, running and sitting [7]. The postural mobility can be either single model or a combination of one or more models.

In the WBAN, the topology may completely change because of posture changes and movement even within a certain type of posture.

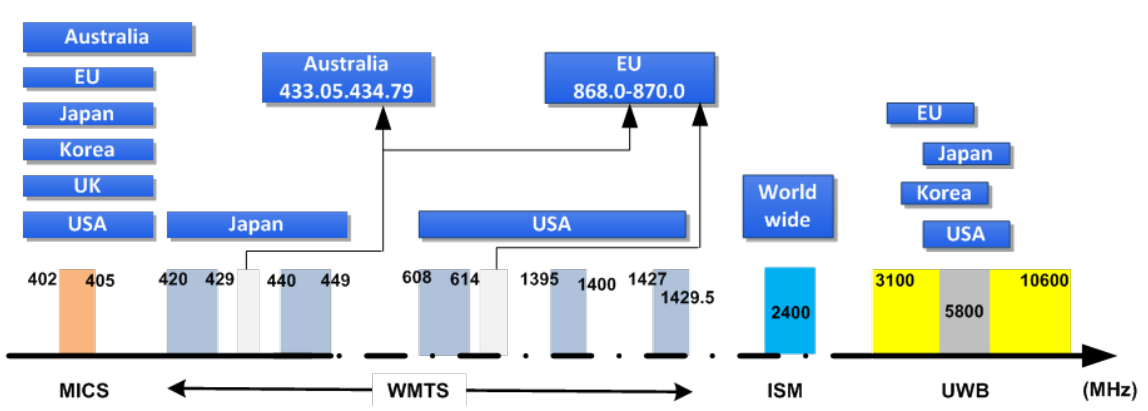

Figure 3. Frequency bands for WBAN [16]. 
In the MoBAN mobility model, we specify the distribution for the time duration of each posture according to the application scenario. This can be a constant time duration or a uniform distribution, or a more precise distribution closer to real-life posture duration.

\section{Related Work}

The CSMA/CA based protocol in WLAN has been a focus of many studies. A few performance analysis studies have been carried out on the IEEE 802.15.6 WBAN as summarized below.

In their study, Alam and Hamida [17] investigated the interference mitigation and coexistence strategies proposed in IEEE 802.15.6 standard within the context of co-channel interference. They investigated the Scheduled access and CSMA/CA MAC protocols for on-body and body-to-body communication. They did a comparative evaluation of the reference scenario (which does not use any coexistence scheme), Time Shared, Random Channel and CSMA/CA based coexistence schemes on five co-located bodies. They concluded that there was trade-off between coexistence schemes. The Time Shared and Random Channel provided much better packet reception ratio and energy efficiency, though they suffered in meeting the delay constraints of the IEEE 802.15.6 standard. The CSMA/CA based approach was able to achieve the delay requirements; however, it did not perform well both in terms of packet reception ratio and energy consumption. There was no network bandwidth efficiency considerations in these mechanisms.

Yang et al. [18] evaluated the effect of user priorities (UPs) on the performance of IEEE 802.15.6 CSMA/CA channel access mechanism in narrow band. Simulation metrics mainly focused on the normalized throughput and average packet delay in which the traffic arrival rate and traffic distribution vary. In addition, they made a performance comparison with the non-priority CSMA/CA which concluded that the IEEE 802.15.6 with user priorities performs better in specific situation. The tested mechanisms also were not tested for high mobility and multi-WBAN interfering scenarios. There was no network bandwidth efficiency considerations in these mechanisms.

Zhang et al. [19] analyzed the transmission delay of IEEE 802.15.6 CSMA/CA mechanism under duty-cycle. They first formulated the expressions of the random delay that a sensor node spends on transmitting packets under asynchronous duty-cycling protocol of IEEE 802.15.6 CSMA/CA. They derived the probabilistic characteristics in terms of the expectation and variance of the delay. They also conducted elaborate simulations to demonstrate the correctness of the theoretical analysis. They concluded that there is need to design and optimize adaptive duty-cycling protocols for WBANs taking into account the tradeoff between the delay and energy consumption.

Hend et al. [20] evaluated the performances of the standard IEEE 802.15.6 when applied on different sensors from CANet eHealth project. CANet was one 
of the projects aimed at embedding a WBAN into a cane to monitor elderly/patients. They evaluated the CSMA/CA access mechanism according to three metrics: Data packet breakdown, latency and energy consumption. The results showed that the standard IEEE 802.15.6 did not perform well on the elderly/patients. This test scenario considered the required WBAN mobility scenario and showed much is needed to improve on the standard IEEE 802.15.6.

In an evaluation case study of IEEE 802.15.6, Al-Mazroa \& Rikli evaluated the Performance evaluation of IEEE 802.15.6 MAC in WBANs. Their objective was to combine various MAC techniques in an adaptive way that takes into consideration the types of sensors, their number, and their traffic and energy requirements. The performance of the proposed techniques were evaluated using some of the standard performance measures such as throughput, delay and energy consumption. Their results showed that the performance of a specific WBAN is affected by varying the slot distribution and the traffic intensity among the various MAC access techniques used in IEEE 802.15.6.

In their study, Kaitalidou et al. [21] looked into the performance assessment of the WBAN standards proposed by the institute of electrical and electronics engineers (IEEE), namely IEEE 802.15.4 and IEEE 802.15.6, emphasizing on the different carrier sensing multiple access/collision avoidance (CSMA/CA) mechanisms. They looked at the packet transmission success rate and energy consumption in their evaluation. They found that due to the different CSMA/CA mechanisms employed, IEEE 802.15.6 is more vulnerable to interference than IEEE 802.15.4 as the transmission power increases. However, in the low transmission power regime, IEEE 802.15.6 outperforms IEEE 802.15.4 in terms of successful reception probability. They concluded that the trade-offs between these two should be seriously taken into consideration when designing a WBAN.

\section{Motivation}

The main objective of proposed model is to make IEE 802.15.6 WBAN more reliable by reduction of WBAN network latencies, improve the bandwidth efficiency and network throughput.

By introduction of CSMA/CA contention window approach, we avoid packet collision during sensor data transmission hence minimizing the number of retransmissions. Under the $\mathrm{CW}$, the model detects when a channel is engaged/busy or idle. When the transmission channel is idle, its counter is decremented to minimum (CWmin) according to the traffic priority CWmin value but when the channel is busy, no packet is transmitted and the counter increments (doubles back off counter) until CWmax and the next channel is scanned for availability.

We have also introduced the idea of priority queues in the proposed model to minimize the number of participants in contention of channel access. Hence, the probability of collision amongst the nodes is diminished. Each node in a WBAN is given a priority value with which it uses to access a channel. High priority 
node traffic are always given first priority for channel access. All these ensures data reliability, efficiency and high performance of the WBAN network.

\section{Proposed Model}

The proposed WBAN interference mitigation model is a hybrid model which employs two approaches, a collaborative CSMA/CA's Contention Window (CW) technique and a priority queue approach.

The approach of priority queues involves the use of User Priorities (UP) mappings in traffic prioritization. This means assigning the sensor nodes traffic different priorities with emergency traffic being assigned high priority and the rest lower priority.

We proposed eight (8) types of priority mappings as shown on Table 2. The higher the user priority value the critical the traffic is. The different User Priority (UP) are assigned the CWmin and CWmax parameters to determine their transmission priority. These CWmin and CWmax are shown in Table 3.

You will note that the smaller the CW value, the easier the nodes accesses the channel for transmission, consequently the bigger the UP the higher the transmission priority for that specific WBAN sensor node. This means a sensor node with highest UP of 7 has the highest priority for channel access and that the traffic in this node has the smallest value of $\mathrm{CW}$ as the nodes with lesser $\mathrm{CW}$ accesses the channel earliest while node with highest CW accesses last.

The benefits of our proposed hybrid model CSMA/CA CW and Priority queues are.

- By use of priority schedules, we can allocate the emergency critical sensor node data the highest priority and the rest of data lower priorities accordingly.

- This model avoids packet collision hence minimising the number of retransmissions by means of Contention Window. Under the CW, the model detects when a channel is busy or idle. When the transmission channel is idle, its counter is reduced to minimum (CWmin) according to the traffic priority CWmin value but when the channel is busy, no packet is transmitted and the counter increments (doubles back off counter) until CWmax and the next channel is scanned for availability.

- Furthermore, the priority queues introduced in the proposed model minimizes the number of participants in contention of channel access. Hence, the probability of collision amongst the nodes is diminished. Each node in a WBAN is given a priority value with which it uses to access a channel. High priority node traffic are always given first priority for channel access. Condolences.

Flow Chart 1 shows the proposed model.

\section{Performance Parameters}

Important notations and parameters list is given in Table 4 and Table 5 respectively. 


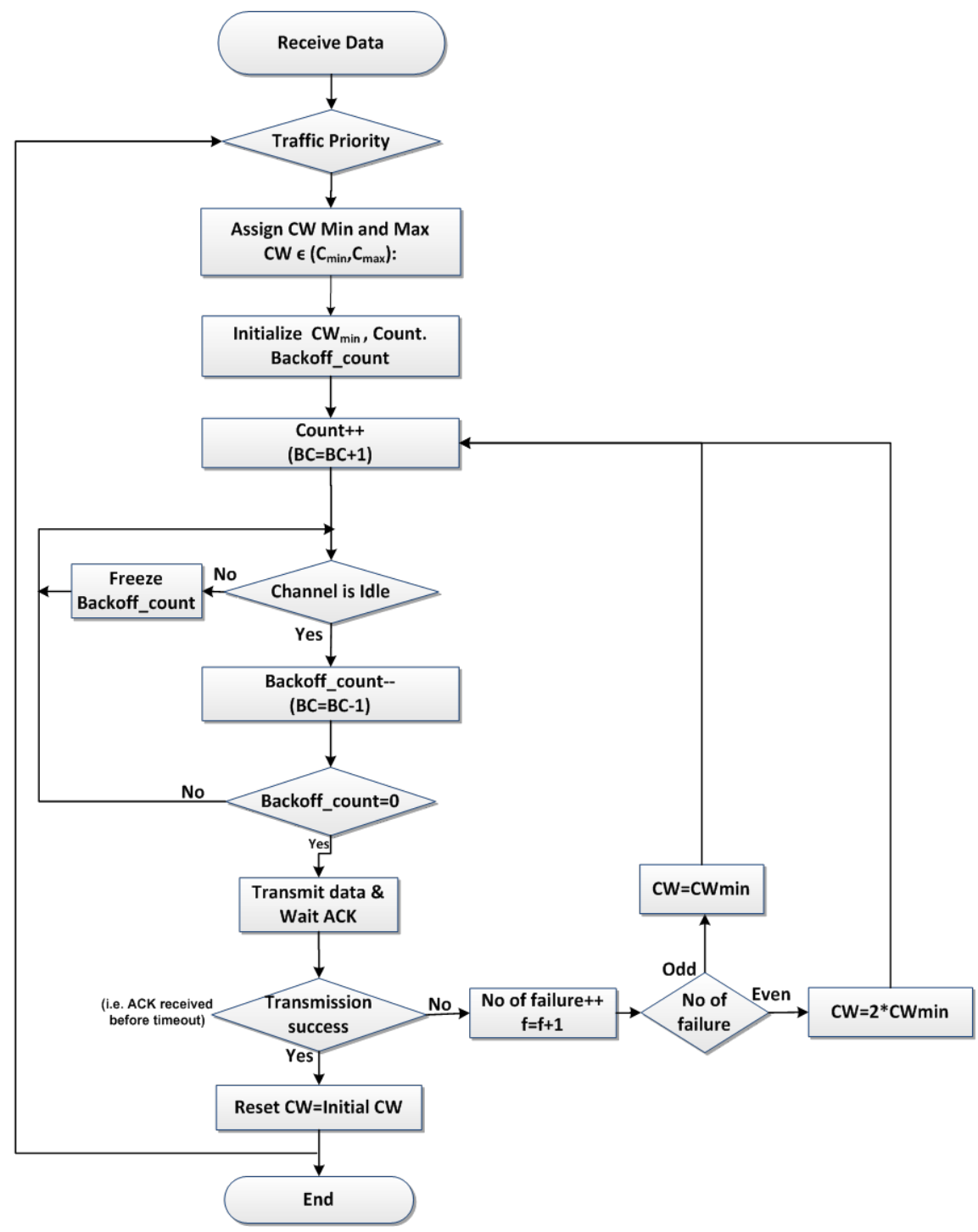

Flow Chart 1. Proposed model.

Table 2. User Priority (UP) frame types.

\begin{tabular}{ccc}
\hline User priority & Traffic Designation & Frame Type \\
\hline 0 & Background (BK) & Data \\
1 & Best effort (BE) & Data \\
2 & Excellent effort (EE) & Data \\
3 & Video (VI) & Data \\
4 & Voice (VO) & Data \\
6 & Medical data or network control & Data or management \\
7 & Emergency or medical implant event report Data & \\
\end{tabular}


Table 3. User priority (UP) $\mathrm{CW}_{\min }$ and $\mathrm{CW}_{\max }$ parameters.

\begin{tabular}{cccc}
\hline User priority & Traffic Type & $\mathrm{CW}_{\min }$ & $\mathrm{CW}_{\max }$ \\
\hline 0 & Background (BK) & 16 & 64 \\
1 & Best effort (BE) & 16 & 32 \\
2 & Excellent effort (EE) & 8 & 32 \\
3 & Video (VI) & 8 & 16 \\
4 & Voice (VO) & 4 & 16 \\
5 & Medical data or network control & 4 & 8 \\
7 & High-priority medical data or network control & 2 & 8 \\
\hline
\end{tabular}

Table 4. Notations used in equations.

\begin{tabular}{cc}
\hline$T_{p}$ & Preamble transmission time \\
$R_{s}$ & Preamble transmission symbol rate \\
$T_{\mathrm{PHY}}$ & PHY layer header transmission time \\
$R_{\mathrm{hdr}}$ & Header rate of PHY layer \\
$R_{\mathrm{DATA}}$ & Transmission rate of data \\
$T_{\mathrm{s}}$ & Slot length \\
$T_{\mathrm{pSIFS}}$ & Short inter-frame spacing time \\
MHR & Mac header \\
FTR & Mac footer \\
$\tau$ & Propagation time \\
$x$ & Payload Size \\
$C W_{\text {min }}$ & Contention Window \\
\hline
\end{tabular}

Table 5. Simulation parameters.

\begin{tabular}{cc}
\hline Parameter Name & Value \\
\hline Saturation & $-90 \mathrm{~dB} B_{m}$ \\
Alpha & 3 \\
Carrier Frequency & $2.412 \mathrm{GHz}$ \\
Time $R_{x}$ to $T_{x}$ & $0.00021 \mathrm{~s}$ \\
Time $R_{x}$ to Sleep & $0.000031 \mathrm{~s}$ \\
Time $T_{x}$ to $R_{x}$ & $0.00012 \mathrm{~s}$ \\
Time $T_{x}$ to Sleep & $0.000032 \mathrm{~s}$ \\
Time Sleep to $R_{x}$ & $0.000102 \mathrm{~s}$ \\
Time Sleep to $T_{x}$ & $0.000203 \mathrm{~s}$ \\
\hline
\end{tabular}




\section{Continued}

\begin{tabular}{cc}
\hline Battery Voltage & $3.3 \mathrm{~V}$ \\
$T_{\text {pMIFs }}$ & $20 \mu \mathrm{s}$ \\
$\mathrm{T}_{\mathrm{pSIFS}}$ & $50 \mu \mathrm{s}$ \\
$\mathrm{T}_{\mathrm{p}}$ & $88 \mathrm{bit} / R_{\mathrm{s}}$ \\
$\tau$ & $1 \mu \mathrm{s}$ \\
$T_{\text {PHY }}$ & $31 \mathrm{bits} / R_{\mathrm{hdr}}$ \\
Mac Queue Length & 5 \\
Slot Duration & $0.00035 \mathrm{~s}$ \\
Max $T_{x}$ Attempts & 14 \\
$R_{\text {DATA }}$ & $187500 \mathrm{bps}$ \\
$C W_{\text {min }}$ & 16 \\
$T_{x}$ Power & $1 \mathrm{~mW}$ \\
MHR & 56 \\
FTR & 16 \\
$R_{s}$ & 187500 \\
Pdelay & 0.000001 \\
$x$ & 2000 \\
\hline &
\end{tabular}

Formula used to calculate delay normally, is given in Equation (1) is calculated in Shakir et al., 2016 [8] as follows;

$$
\text { Delay }(x)=T_{\text {avg_backoff }}+T_{\text {DATA }}+T_{\text {iack }}+2 T_{\text {pSIFS }}+2 \tau
$$

The average back off time can be found as shown in Equation (2);

$$
T_{\text {avg_backoff }}=\frac{C W_{\min } \times T_{s}}{2}
$$

The transmission time of data is $T_{\text {DATA }}$ and can be obtained as in Equation (3);

$$
T_{\mathrm{DATA}}=T_{\mathrm{p}}+T_{\mathrm{PHY}}+\frac{(\mathrm{MHR}+x+\mathrm{FTR})}{R_{\mathrm{DATA}}}
$$

The transmission time of immediate acknowledgement can be obtained as in Equation (4);

$$
T_{\text {iack }}=T_{\mathrm{P}}+T_{\mathrm{PHY}}+\frac{((\mathrm{MHR}+\mathrm{FTR}) * n)}{R_{\mathrm{DATA}}}
$$

Maximum Throughput (MT) of network is directly related to overhead.

The MT is defined as the ratio of payload size (x) to the total transmission delay per payload size Delay (x), as given below in Equation (5) [8];

$$
M T=\frac{x}{\operatorname{Delay}(x)}
$$


Maximum Throughput (MT) in case of immediate acknowledgement can be found by using Equation (6);

$$
M T_{\text {iack }}=\frac{x}{\left(T_{\text {avg_backoff }}+T_{\text {DATA }}+T_{\text {iack }}+2 T_{\text {pSIFS }}+2 \tau\right)}
$$

The bandwidth efficiency is inversely proportional to the basic data rate as in Equation (7);

$$
\rho=\frac{M T}{R_{\mathrm{DATA}}}
$$

Hence as a percentage ratio this becomes:

$$
\rho=\frac{M T}{R_{\mathrm{DATA}}} * 100
$$

\section{Performance Analysis}

We have evaluated the performance of the proposed hybrid WBAN interference mitigation model under varying postural mobility scenarios of lying down, walking, standing and running and compared with the standard IEEE 802.15.6 WBAN using bandwidth efficiency, network throughput and network delay as main performance metrics. This is unique testing as no previous research has really evaluated these under the different mobility scenarios.

In the following result diagrams, the dotted lines represent the proposed model (PH1) while the continuous line graphs represent the existing model (H1). The walking posture is represented in colour red, the lying down posture is represented by colour green, the sitting posture is represented by color blue while the standing is represented by colour pink.

In the first diagram, Figure 4, we show the mean bandwidth efficiency of walking, lying down, sitting and standing postural nobilities against time for both the existing standard (abbreviated by $\mathrm{H} 1$ ) and the proposed CSMA/CA CW (abbreviated by $\mathrm{PH} 1)$. The results show that the proposed model (PH1) has a significant improvement in bandwidth efficiency under all the scenarios of walking, lying down, sitting and standing postural mobility from an average performance of $50 \%$ to $80 \%$ efficiency. The results diagram shows the lower level line graphs as $\mathrm{H} 1$ while the proposed (PH1) related line graphs being higher in the efficiency graph hence signaling improvement in the proposed.

We define the throughput as the sum of the number of successful messages delivered per a unit time at a node. Figure 5 shows the performance of the existing IEEE 802.15.6 WBAN model (H1) and the proposed WBAN interference mitigation model (PH1) against time for the network throughput. The dotted lines indicating the proposed model confirms an improvement in bandwidth efficiency of the proposed by a significant level in mobility scenarios. The lying posture shows the most performing posture model which can be attributed to the minimal mobility nature of the posture which basically experiences nodal mobility only with group mobility being constant. The results show increased 
network throughput in the proposed model for a WBAN for all the postural mobility scenarios from average of $80 \mathrm{Kbps}$ to $150 \mathrm{Kbps}$.

Network delay is an important factor in the performance of WBAN because of the nature of the applicability of the WBAN. In our simulation results, the network delay performance is demonstrated in Figure 6, in which the delay is shown against time for the various postural mobility. The proposed model (dotted) showed improved performance in all mobility scenarios as compared to the existing (continuous line graphs). We see a decrease in network delay in the proposed interference mitigation model $(\mathrm{PH} 1)$ for all postures compared to the existing IEEE 802.15.6 WBAN (H1). This indicates a magnificent improvement in the WBAN in terms of delay performance. This a clear demonstration of the improvement in the proposed model.

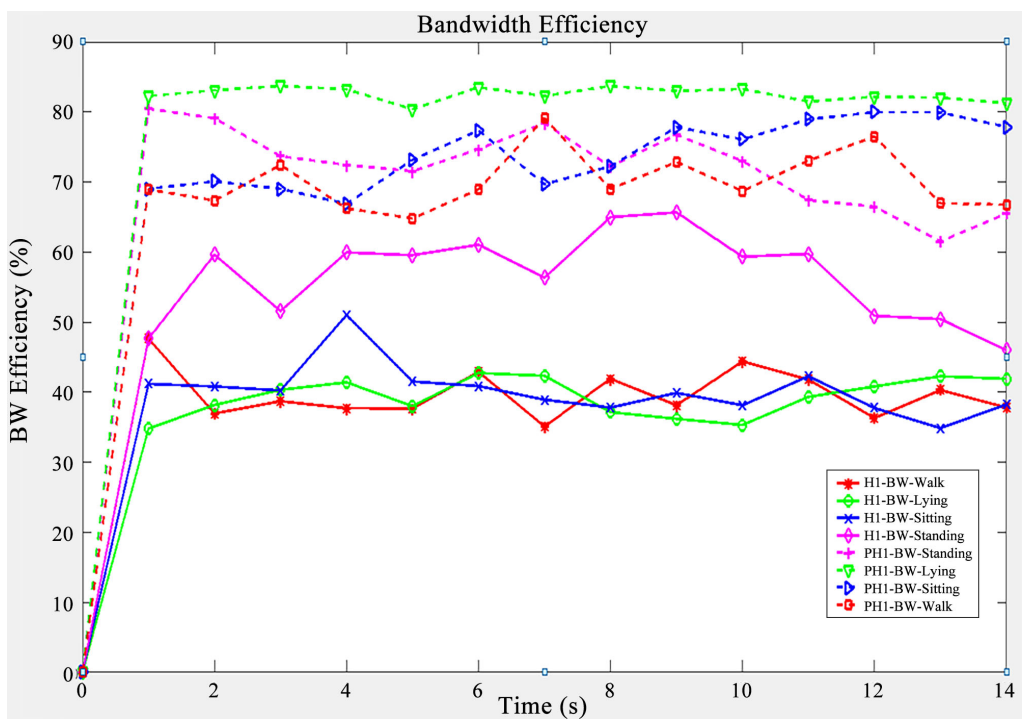

Figure 4. WBAN Bandwidth efficiency Comparison results.

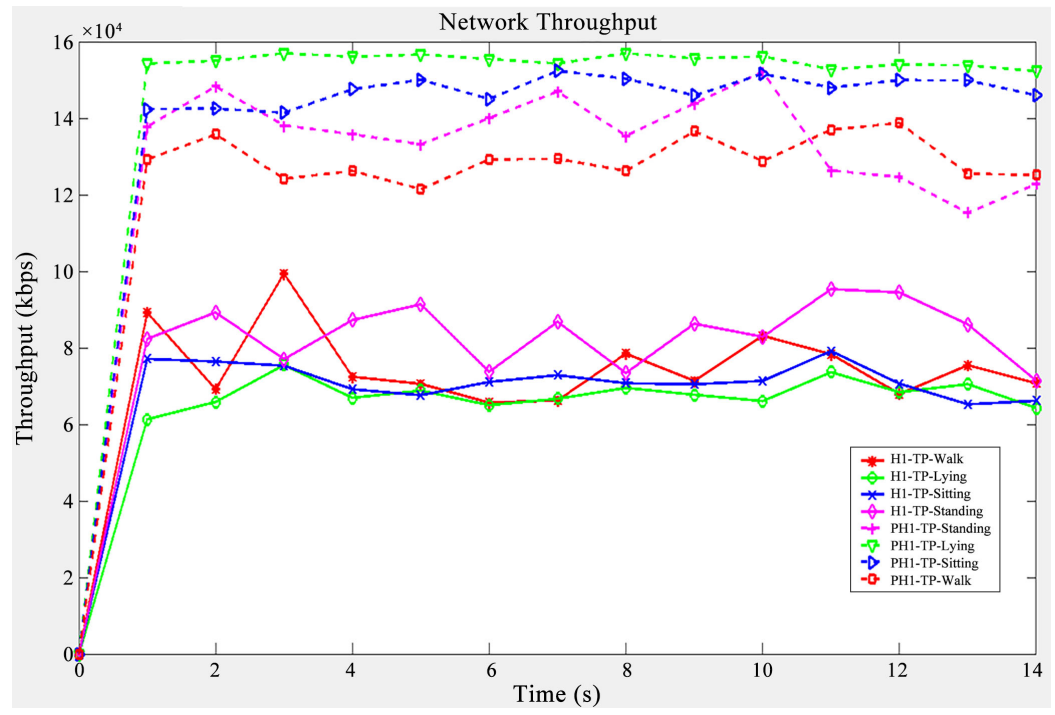

Figure 5. Throughput results of the IEEE 802.15.6 standard. 


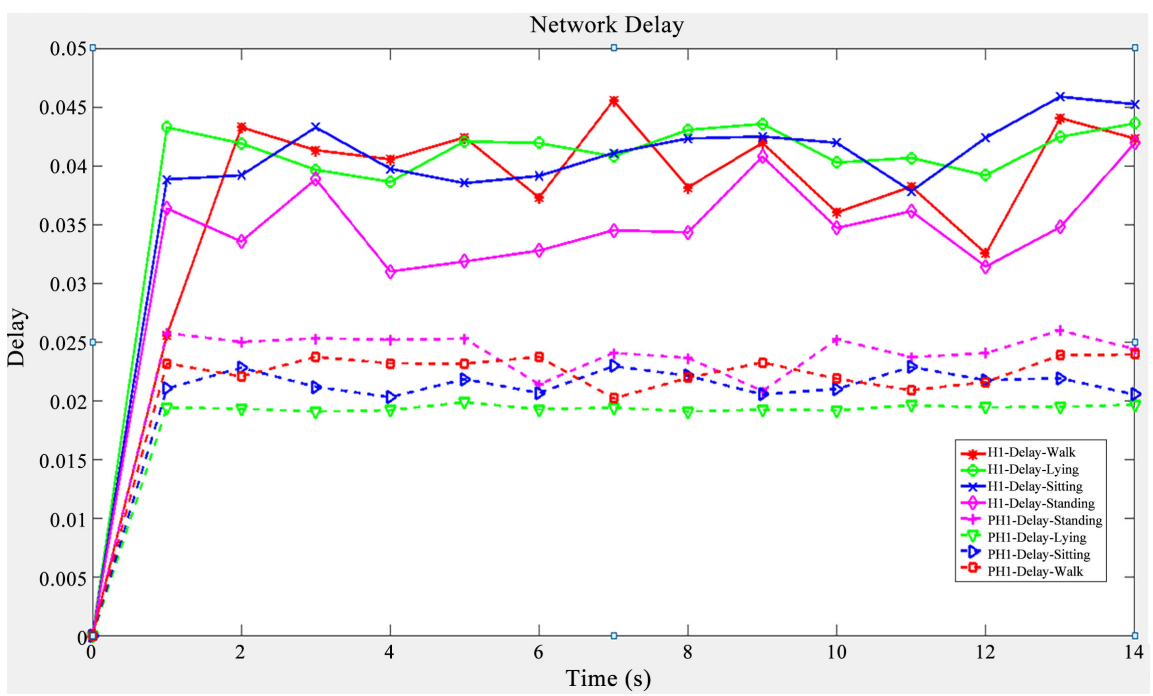

Figure 6. Network delay results of the base IEEE 802.15.6 standard for various WBAN.

\section{Conclusion}

The WBAN effectiveness is very important in the adaptation of remote medical health monitoring for ubiquitous and affordable healthcare. The standard minimum performance throughput is indicated to be $10 \mathrm{kbps}$ with a maximum of 10 Mbps while error rate should be less than $10 \%$ (meaning that the efficiency should be high) hence the need for continuous improvement of the WBAN technologies performance especially in high WBAN interference operating environments. Basing on the results from the different mobility scenarios, our proposed model outperforms the existing IEEE 802.15.6 based WBAN in all the areas of network delay, bandwidth efficiency and network throughput. With bandwidth efficiency improvement from average of $50 \%$ to $80 \%$, network throughput improvement from $70 \mathrm{Kbps}$ to $160 \mathrm{Kbps}$ and network delay reduction from average of $45 \mathrm{~ms}$ to $20 \mathrm{~ms}$, we conclude that the proposed interference mitigation model is a significant contribution to the improvement of the IEEE 802.15.6 based wireless body area networks deployed in mobility scenarios.

\section{References}

[1] Akyildiz, I. and Kasimoglu, I. (2004) Wireless Sensor and Actor Networks: Research Challenges. Ad Hoc Networks, 2, 351-367. https://doi.org/10.1016/j.adhoc.2004.04.003

[2] IEEE STANDARD (2015) IEEE 802.15.4-2015 - IEEE Standard for Low-Rate Wireless Networks.

[3] Ghamari, M., Janko, B., Sherratt, R. S., Harwin, W., Piechockic, R. and Soltanpur, C. (2016) A Survey on Wireless Body Area Networks for Ehealthcare Systems in Residential Environments. Sensors, 16, 831. https://doi.org/10.3390/s16060831

[4] IEEE Standard (2012) IEEE Standard for Local and Metropolitan Area Networks Part 15.6: Wireless Body Area Networks, 1-271.

[5] Yang, G.-Z. (2006) Body Sensor Networks. Springer-Verlag London Limited. https://doi.org/10.1007/1-84628-484-8 
[6] Varga, A. (1999) Using the OMNeT++ Discrete Event Simulation System in Education. IEEE Transactions on Education, 42, 11 p. https://doi.org/10.1109/13.804564

[7] Nabi, M., Geilen, M. and Basten, T. (2011) MoBAN: A Configurable Mobility Model for Wireless Body Area Networks. Proceedings of the 4th International ICST Conference on Simulation Tools and Techniques, ICST (Institute for Computer Sciences, Social-Informatics and Telecommunications Engineering), 168-177. https://doi.org/10.4108/icst.simutools.2011.245511

[8] Shakir, M., Rehman, O.U., Rahim, M., Alrajeh, N., Khan, Z.A., Khan, M. and Javaid, N. (2016) Performance Optimization of Priority Assisted CSMA/CA Mechanism of 802.15. 6 under Saturation Regime. Sensors, 16, 1421. https://doi.org/10.3390/s16091421

[9] Lewis, D. (2010) IEEE p802. 15.6/d0 Draft Standard for Body Area Network (Vol. 6). 15-10-0245-06.

[10] IEEE Draft (2010) IEEE p802.15.6/d0 Draft Standard for Body Area Network.

[11] Zhen, B., Patel, M., Lee, S., Won, E. and Astrin, A. (2008) TG6 Technical Requirements Document (TRD). IEEE P802, 15-08.

[12] Zhang, A., Smith, D., Miniutti, D., Hanlen, L., Rodda, D. and Gilbert, B. (2010) Performance of Piconet Co-Existence Schemes in Wireless Body Area Networks. IEEE Wireless Communications and Networking Conference (WCNC), 1-6. https://doi.org/10.1109/WCNC.2010.5506746

[13] Hanlen, L., Miniutti, D., Smith, B., Rodda, D. and Gilbert, B. (2010) Co-Channel Interference in Body Area Networks with Indoor Measurements at $2.4 \mathrm{GHz}$ : Distance-to-Interferer Is a Poor Estimate of Received Interference Power. IJWIN, 17, 113-125. https://doi.org/10.1007/s10776-010-0123-Z

[14] Movassaghi, S., Abolhasan, M., Lipman, J., Smith, D. and Jamalipour, A. (2014) Wireless Body Area Networks: A Survey. IEEE Communications Surveys \& Tutorials, 16, 1658-1686. https://doi.org/10.1109/SURV.2013.121313.00064

[15] Sana, U., Manar, M. and Mohammed, A. (2013) A Review of IEEE 802.15.6 MAC, PHY, and Security Specifications. International Journal of Distributed Sensor Networks, 2013, Article ID: 950704.

[16] Arthur, A., Huan, L. and Ryuji, K. (2009) Standardization for Body Area Networks. IEICE Transactions on Communications, E92.B, 366-372.

[17] Alam, M.M. and Hamida, E.B. (2016) Performance Evaluation of IEEE 802.15. 6-Based WBANs under Co-Channel Interference.

[18] Yang, L., Li, C., Song, Y., Yuan, X. and Lei, Y. (2015) Performance Evaluation of IEEE 802.15. 6 MAC with User Priorities for Medical Applications. In: Future Information Technology-II, Springer, Dordrecht, 233-240.

[19] Zhang, R., Moungla, H. and Mehaoua, A. (2015) Delay Analysis of IEEE 802.15. 6 CSMA/CA Mechanism in Duty-Cycling WBANs. Global Communications Conference (GLOBECOM), 2015 IEEE, 1-6.

[20] Hend, F., Hanen, I., Thierry, V., Adrien, V. and Leila, A. (2015) IEEE International Conference on Computer Systems and Applications (AICCSA).

[21] Kaitalidou, D.S., Boulogeorgos, A.A.A. and Pavlidou, F.N. (2016) Comparison of CSMA/CA Protocols Applied in Wireless Body Area Network Standards. Electrotechnical Conference (MELECON), 2016 18th Mediterranean, IEEE, 1-6. 